15

\title{
Определение температуропроводности материалов методом нестационарного точечного нагрева
}

\author{
() Д.Ю. Головин ${ }^{1}$, А.И. Тюрин ${ }^{1}$, А.А. Самодуров ${ }^{1}$, Ю.И. Головин ${ }^{1,2, \text { Ф }}$ \\ ${ }^{1}$ Научно-исследовательский институт „Нанотехнологии и наноматериалы“ Тамбовского государственного университета \\ им. Г.Р. Державина, Тамбов, Россия \\ ${ }^{2}$ Московский государственный университет им. М.В. Ломоносова, Москва, Россия \\ ๑E-mail: yugolovin@yandex.ru
}

Поступило в Редакцию 30 сентября 2019 г.

В окончательной редакции 30 сентября 2019 r.

Принято к публикации 4 октября 2019 г.

\begin{abstract}
Описан термографический экспресс-метод определения коэффициента температуропроводности $a$ пластинчатых изделий, который можно рассматривать как модификацию и дальнейшее развитие метода лазерной вспышки, при этом в отличие от последнего предложенный метод не требует вырезки образцов и двустороннего доступа к объекту. Он заключается в точечном ступенчатом нагреве объекта сфокусированным лазерным пучком $\left(\sim 0.1 \mathrm{~mm}^{2}\right)$ с одновременной высокоскоростной регистрацией картины нестационарного температурного поля тепловизором. Разработанный алгоритм извлечения величины $a$ из первичных данных исключает необходимость определения абсолютных температур и мощности поглощенной энергии падающего потока энергии.
\end{abstract}

Ключевые слова: температуропроводность, нестационарный точечный нагрев, тепловизор.

DOI: 10.21883/PJTF.2020.01.48863.18052

Знание теплофизических характеристик (ТФХ) материалов и изделий необходимо при проектировании и эксплуатации энергетического оборудования, мощной лазерной техники, наземных и аэрокосмических транспортных средств, оборудования химических производств, радио- и электротехнических изделий и др. Известны две большие группы методов измерения ТФХ: стационарные и нестационарные [1-5]. Первые в принципе не позволяют измерять прямыми способами коэффициент температуропроводности $a$, необходимый для расчета распространения теплового фронта в условиях импульсного нагрева изделия. Среди нестационарных способов большое распространение получил метод лазерной вспышки (МЛВ), принципы которого предложил У. Паркер с соавторами еще в 1961 г. [6]. С тех пор этот метод значительно усовершенствовался и вошел в международные стандарты [7-9], но сохранил неустранимые недостатки: необходимость вырезки образца определенной формы и размеров и двустороннего доступа к нему. Эти особенности делают МЛВ весьма трудоемким, малопроизводительным и снижающим выпуск готовой продукции.

В работе предложена экспресс-модификация МЛВ, не требующая вырезки образцов из изделия, двустороннего доступа к нему и подготовки поверхности к испытанию. Она заключается в точечном ступенчатом нагреве поверхности объекта сфокусированным лазерным пучком (с гауссовым радиусом $r_{0}=0.1-0.3 \mathrm{~mm}$ ) с одновременной высокоскоростной регистрацией тепловизором картины нестационарного температурного поля (рис. 1). Особенностью способа, поддерживаемого оригинальным алгоритмом обработки первичных данных и разработан- ным программным обеспечением, являются использование радиальной симметрии картины изотерм (на поверхности однородного материала) и накопление за время теста, занимающего единицы-десятки секунд, большого массива первичных данных (10-100 MB). Усреднение сигналов по такому массиву данных позволяет существенно увеличить отношение сигнал/шум и таким образом обеспечить среднеквадратическое отклонение конечного результата в $2-4 \%$.

Опытный образец портативного прибора содержал тепловизор FLIR A35sc, установленный на направляющих, снабженных датчиком продольного перемещения Vogel-150 (для изменения поля зрения и точного масштабирования изображения). Точечный нагрев осуществлялся $\mathrm{Nd}$ : YAG-лазером (лазерный модуль LSR445CP-FC-10W с регулируемой непрерывной мощностью излучения от 1 до $10 \mathrm{~W}$ ), снабженным коллиматором. Лазер не создавал короткую вспышку, как в МЛВ и его латеральных (in plane) модификациях [10], a paботал непрерывно в течение всего процесса измерения. Это позволяло значительно уменьшить локальный перегрев в центре пятна нагрева, одновременно увеличив температуру во всей инспектируемой области, а также снизить требования к мощности лазера. Тепловизор мог располагаться как на одной стороне с лазером, так и с противоположной от него (см. вставку $a$ на рис. 1), что не вносит принципиальных различий в существо метода, а приводит лишь к небольшим техническим изменениям в реализации. Кадры фильма преобразовывались в двумерные карты распределения температуры. Для уменьшения влияния неоднородности оптических свойств поверхности образца из каждой такой карты 


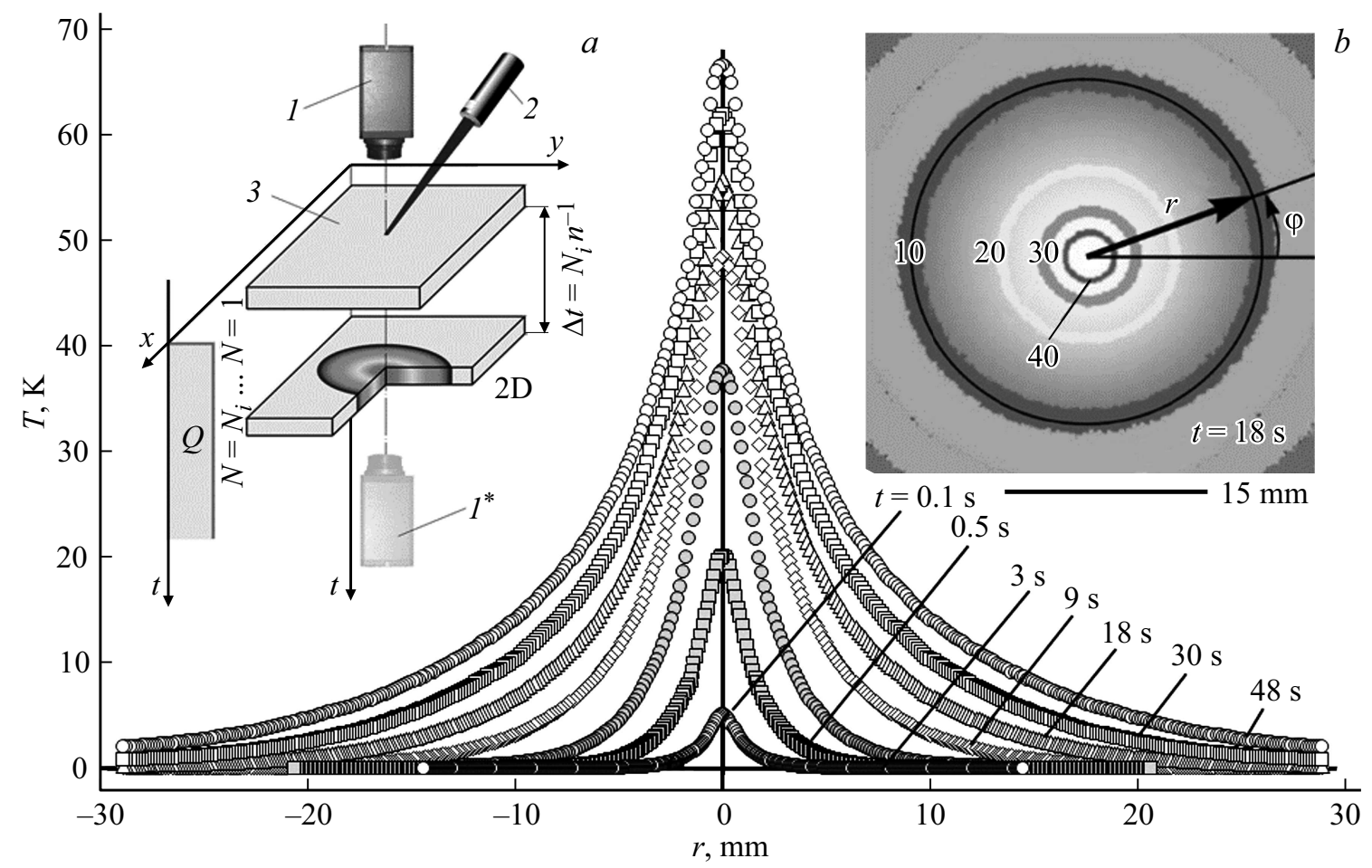

Рис. 1. Экспериментальная зависимость температуры $T$ от расстояния до центра пятна нагрева $r$ в листе нержавеющей стали $12 \mathrm{X} 18 \mathrm{H} 9 \mathrm{~T}$ толщиной $\delta=1 \mathrm{~mm}$ для разных моментов времени $t$. На вставке $a$ приведена схема эксперимента: 1 и $1^{*}-$ тепловизор и его положение при реализации одностороннего и двустороннего тестов соответственно, $2-$ лазер, 3 - исследуемый образец; $n=10-60 \mathrm{~s}^{-1}-$ скорость кинофильмирования, $N-$ номер кадра. На вставке $b-$ пример отдельного кадра термограммы со схемой усреднения температуры (в К) по углу $\varphi$.

попиксельно вычиталась карта начального состояния до начала нагрева. В последующем оперировали величиной разницы $T=T_{h}-T_{0}$, где $T_{h}-$ мгновенная локальная температура, $T_{0}$ - температура перед испытанием. Затем определялось положение центра симметрии изотерм и производилось усреднение величины $T$ по углу $\varphi$ до $360^{\circ} \mathrm{C}$ (вставка $b$ на рис. 1) при наборе фиксированных расстояний $r$ от центра пятна нагрева, что сильно снижало шумы и помехи при измерениях. Для определения $a$ в однородных изотропных материалах было принято $\varphi=360^{\circ} \mathrm{C}$. Верификация метода проведена на пластинах толщиной $b=1-2 \mathrm{~mm}$ из трех металлических материалов: стали Ст 35 , нержавеющей стали 12 Х18Н9Т и титанового сплава ОТ4-2, различающихся теплофизическими и оптическими свойствами поверхности.

Кратко опишем расчетную модель извлечения из этих данных величины $a$. В однородной пластине точечный нагрев генерирует радиально симметричное тепловое поле $T(r, t)$. Оно может быть описано наиболее простым образом, когда тепловой фронт распространится от центра пятна нагрева на расстояние $r \gg r_{0}$ и $r \gg b$, а картины изотерм на передней и тыльной поверхностях стенки станут практически одинаковыми и симметрия поля приобретет цилиндрический характер. Для этого необходимо выполнение двух условий:

$$
\mathrm{Fo}_{b}=t a / b^{2} \gg 1, \quad \mathrm{Fo}_{r_{0}}=t a / r_{0}^{2} \gg 1,
$$

где $\mathrm{Fo}_{b}$ и $\mathrm{Fo}_{r_{0}}$ - критерии Фурье, соответствующие размерам $b$ и $r_{0}$. Тогда при нагреве тепловым потоком $Q$ поле $T(r, t)$ в пластине может быть описано выражением [11]:

$$
T(r, t)=(Q / 4 \pi \lambda \delta) \ln \left(4 a t / C r^{2}\right)+O\left(r_{0}^{2} / a t\right),
$$

где $\ln C=\gamma \approx 0.5772$ - постоянная Эйлера, $O(x)-$ некоторая функция, лимитированная значением своего аргумента, умноженного на конечную константу. При выполнении условий (1) вторым членом в (2) можно пренебречь, и $T(r, t)$ будет расти со временем логарифмически. В принципе для вычисления $а$ достаточно измерения двух значений $T$ при $r_{1}, t_{1}$ и $r_{2}, t_{2}$, что обычно и делают в методе горячей проволоки $[12,13]$ с точечными сенсорами температуры. Однако, располагая большим объемом данных о динамике поля температур, величину $a$ можно получить гораздо более точным способом - путем линейной регрессии функции $y=d(x+g)$ (рис. 2). Здесь $d-$ наклон аппроксимирующей прямой, $g$ - отрезок, отсекаемый ею на оси абсцисс в координатах $T-\ln \left(t r^{-2}\right)$. Тогда в соответствии с выражением (2) искомая величина $a$ может быть найдена с помощью выражения

$$
a=\exp (g+\gamma) / 4 \text {. }
$$




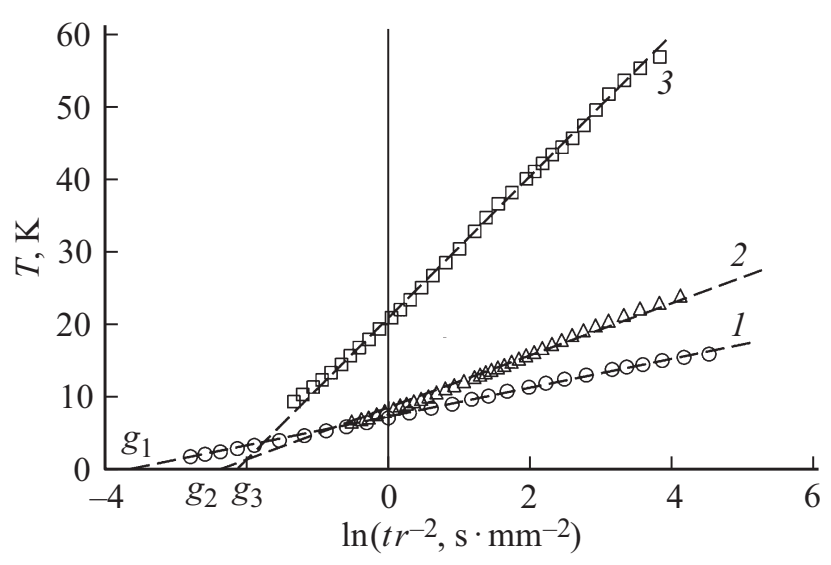

Рис. 2. Экспериментальные данные (точки) и их линейные аппроксимации (штриховые линии) при 2D-испытаниях для стали Ст 35 при $t=40 \mathrm{~s}(1)$, титанового сплава при $t=48 \mathrm{~s}(2)$ и нержавеющей стали при $t=48 \mathrm{~s}(3) . \mathrm{g}_{1}, g_{2}$ и $g_{3}$ - отрезки, отсекаемые аппроксимирующей прямой на оси абсцисс для стали Ст 35, титанового сплава ОТ4-2 и стали 12Х18Н9Т соответственно.

В результате обработки от 500 до 800 кадров описанным способом для стали Ст 35 получено среднее значение $a=13.37 \pm 0.33 \mathrm{~mm}^{2} / \mathrm{s}$, среднеквадратическое отклонение составило всего $\pm 2.5 \%$. (см. таблицу). Оно хорошо согласуется со значением, полученным по соотношению $a^{*}=\lambda / \rho c_{p}$ исходя из табличных значений материальных констант для стали Ст 35 (плотность $\rho=7796 \mathrm{~kg} / \mathrm{m}^{3}$, удельная теплоемкость $c_{p}=462 \mathrm{~J} /(\mathrm{kg} \cdot \mathrm{K})$ и коэффициент теплопроводности $\lambda=48 \mathrm{~W} /(\mathrm{m} \cdot \mathrm{K})[14])$. Найденное расчетное значение составило $a^{*}=13.33 \mathrm{~mm}^{2} / \mathrm{s}$. Аналогичные данные получены для нержавеющей стали и титанового сплава (см. таблицу).

Модель цилиндрического фронта накладывает ограничения на толщину образца $b$. Снизу оно определяется увеличением роли теплоотвода, характеризуемой от- ношением энергии, покинувшей пластину посредством теплообмена на поверхностях, к запасенной энергии $a \tau / c \rho b$ на единице площади пластины, где $\alpha-$ суммарный коэффициент теплоотдачи (как конвективной, так и радиационной для обеих свободных поверхностей пластины). Увеличение $b$ приводит к пропорциональному росту необходимой мощности источника тепла для разогрева образца до той же температуры и квадратично большему времени для распространения теплового фронта на минимальное расстояние $\sim 10 b$, необходимое для получения точности измерения порядка единиц процентов при использовании модели цилиндрического теплового фронта. Максимальный нагрев в области тестирования зависел от плотности потока в пятне нагрева, его радиуса и длительности процедуры измерения. Ни в каких опытах он не превышал нескольких десятков градусов Цельсия, а в области измерения единиц градусов Цельсия, что позволяет считать метод неразрушающим и относить полученные значения $a$ к температуре окружающей среды.

Подчеркнем, что в предложенном подходе не требуется знания абсолютных значений температур поверхности и тепловых потоков, поскольку они не входят в формулу (3) для вычисления $a$. Достаточно лишь допущения оптической однородности поверхности, а именно постоянства коэффициента испускания.

Итак, в работе предложен новый экспрессный неразрушающий способ определения коэффициента температуропроводности пластинчатых объектов. Экспериментальное тестирование показало, что определенные этим способом значения коэффициентов температуропроводности и теплопроводности с хорошей точностью совпадают со справочными данными. Описанный подход можно рассматривать как дальнейшее развитие МЛВ. Низкая погрешность при невысоких требованиях $к$ условиям измерений в значительной мере обусловлена возможностями анализа не однократно регистрируемой функции одного аргумента $T(t)$ в каждом отдельномопыте, как в МЛВ, а большого ансамбля первичных данных

Полученные и справочные значения $а$ для исследованных материалов

\begin{tabular}{|c|c|c|c|c|c|c|c|}
\hline \multirow{2}{*}{ Материал } & \multirow{2}{*}{$\begin{array}{c}\text { Измеренные и } \\
\text { справочные значения } a \text {, } \\
\mathrm{mm}^{2} / \mathrm{s}\end{array}$} & \multicolumn{6}{|c|}{ Номер теста } \\
\hline & & 1 & 2 & 3 & 4 & 5 & 6 \\
\hline Сталь Ст 35 & $\begin{array}{l}a \\
a_{6} \\
a^{*}\end{array}$ & \multicolumn{6}{|c|}{$\begin{array}{c}13.37 \pm 0.33 \\
13.33\end{array}$} \\
\hline Титановый сплав ОТ4-2 & $\begin{array}{l}a \\
a_{6} \\
a^{*}\end{array}$ & \multicolumn{6}{|c|}{$\begin{array}{c}3.89 \pm 0.15 \\
3.81\end{array}$} \\
\hline Сталь 12Х18Н9Т & $\begin{array}{l}a \\
a_{6} \\
a^{*}\end{array}$ & 3.68 & 3.39 & $\begin{array}{l}3.16 \\
3.5\end{array}$ & $\begin{array}{l}3.60 \\
.10\end{array}$ & 3.97 & 3.45 \\
\hline
\end{tabular}

Пр и ме чан ие. Значения $a^{*}$ получены с помощью соотношения $a^{*}=\lambda / \rho c_{p}$ на основании данных, взятых из работы [14]. 
в виде двухпараметровой матрицы $T(r, t)$ в каждом кадре, а затем - анализа нескольких сотен кадров.

\section{Благодарности}

Работа выполнена с использованием оборудования ЦКП ТГУ им. Г.Р. Державина.

\section{Финансирование работы}

Разработка методики и портативного прибора для определения ТФХ выполнена при поддержке гранта Российского научного фонда (проект № 15-19-00181), a разработка численных моделей и программного обеспечения - при поддержке гранта Российского фонда фундаментальных исследований (проект № 17-48-680817).

\section{Конфликт интересов}

Авторы заявляют, что у них нет конфликта интересов.

\section{Список литературы}

[1] Сквориов Л.А. Основы фототермической радиометрии и лазерной термографии. М.: Техносфера, 2017. 220 с.

[2] Методы определения теплопроводности и температуропроводности / Под ред. А.В. Лыкова. М.: Энергия, 1973. $336 \mathrm{c}$.

[3] Вавилов В.П. Инфракрасная термография и тепловой контроль. М.: Спектр, 2013. 542 с.

[4] Hammerschmidt U., Hameury J., Strnad R., Turzó-Andras E., Wu J. // Int. J. Thermophys. 2015. V. 36. P. 1530-1544.

[5] Yüksel $N$. The review of some commonly used methods and techniques to measure the thermal conductivity of insulation materials // Insulation materials in context of sustainability / Eds A. Almusaed, A. Almssad. London: InTech, 2016. Ch. 6. P. 113-140.

[6] Parker W.J., Jenkins R.J., Butler C.P., Abbot G.L. // J. Appl. Phys. 1961. V. 32. P. 1679-1684.

[7] ISO 22007-4:2017. Plastics - Determination of thermal conductivity and thermal diffusivity. Pt 4. Laser flash method.

[8] ISO 18755:2005. Fine ceramics (advanced ceramics, advanced technical ceramics) - Determination of thermal diffusivity of monolithic ceramics by laser flash method.

[9] ASTM E1461-13. Standard test method for thermal diffusivity by the flash method.

[10] Kruczek T., Adamczyk W.P., Bialecki R.A. // Int. J. Thermophys. 2013. V. 34. P. 467-485.

[11] Carslaw H.C., Jaeger J.C. Conduction of heat in solids. Oxford University Press, $1959.510 \mathrm{p}$.

[12] Assael M.J., Antoniadis K.D., William A. // Int. J. Thermophys. 2010. V. 31. P. 1051-1072.

[13] Guo W., Li G., Zheng Y., Dong C. // Thermochim. Acta. 2018. V. 661. P. 84-97.

[14] Лариков Л.Н., Юрченко Ю.Ф. Тепловые свойства металлов и сплавов. Справочник. Киев: Наук. думка, 1985. 438 с. 